\title{
Ulnar Claw-Hand Related Neglected Post-Traumatic Anterior Shoulder Joint Dislocation
}

\author{
Hermawan Nagar Rasyid \\ Department of Orthopaedics and Traumatology, Faculty of Medicine, Universitas Padjadjaran, Dr. Hasan Sadikin Teaching \\ Hospital, Bandung, Indonesia \\ Email: hermawanphd@gmail.com
}

How to cite this paper: Rasyid, H.N. (2017) Ulnar Claw-Hand Related Neglected Post-Traumatic Anterior Shoulder Joint Dislocation. Open Access Library Journal, 4: e3454.

https://doi.org/10.4236/oalib.1103454

Received: February 13, 2017

Accepted: March 17, 2017

Published: March 20, 2017

Copyright (c) 2017 by author and Open Access Library Inc.

This work is licensed under the Creative Commons Attribution International License (CC BY 4.0).

http://creativecommons.org/licenses/by/4.0/ (c) (i) Open Access

\begin{abstract}
Shoulder joint is the most frequently dislocated joint. Humeral head dislocation pushed the nerve toward medial side. Neglected shoulder dislocation is difficult to manage and requires extensive procedures to obtain good functional outcome. In the case of negligence, it is often found loss of the anterior capsule due to absorption of the capsule. Nerve lesions, in particular the ulnar nerve, often do not receive attention. Clinically, it often occurred from neurapraxia to severe condition like claw-hand deformity. In my experience of a neglected case, there was a 53-year-old woman who presented to the orthopaedic clinic with a left anterior shoulder fracture dislocation following a fall onto the right shoulder and upper right arm. She had treated herself at home for around six months before visiting the clinic. She also complained of some deformities on her ring and little fingers, known as ulnar claw-hand. Numbness and tingling on her left hand especially on the ring and little fingers were observed. These findings were carefully documented and the patient was aware of these persistent abnormalities. She had electromyography (EMG) and nerve conduction velosity (NCV) examination evidence of persistent ulnar nerve deficit, performed before and after surgery. We may conclude that this case highlights the need for awareness of the potential for nerve damage following shoulder dislocation. After treating the dislocation, the functional and EMG results are very satisfactory.
\end{abstract}

\section{Subject Areas}

Orthopedics

\section{Keywords}

Anterior Shoulder Dislocation, Neglected, Ulnar Claw-Hand 


\section{Introduction}

Ulnar claw-hand is related to neglected shoulder dislocation but is seldom discussed in literature and consequently, there is no consensus regarding its treatment. Post-traumatic anterior shoulder dislocation is the most common type of dislocation and is caused by excessive external rotation in abduction levers, the head of the humerus out of the glenoid socket, avulsing anterior bony and soft tissue structures in the process (the Bankart lesion) [1]. In the end, posterior part of the humeral head exists the joint, and it often collides with anterior rim of the glenoid fossa, creating a bony indentation at the back of the humeral head (the Hill Sachs lesion) [2] [3]. Moreover, anterior shoulder dislocation can be followed by rotator cuff tears, especially in elderly patients, and nerve compression. Failure to reduce a dislocated shoulder successfully within 24 hours carries the risk that it will be impossible to achieve a stable closed reduction [1].

Incidence of brachial plexus injury following anterior dislocation is approximate $8 \%$. In contrast, brachial plexus injury is rare and complicated, and very few cases have been reported in the literature [4]. Ulnar nerve is an extension of the medial cord of the brachial plexus. It is a mixed nerve that supplies innervation to muscles in the forearm and hand and provides sensation over the medial half of the ring finger and entire little finger (ulnar aspect of the palm) and ulnar portion of the posterior aspect of the hand (dorsal ulnar cutaneous distribution) [5]. The ulnar nerve in his travels will control motion and sensation as well in some of the muscles of arm and hand. Pressure on or injury to the ulnar nerve may cause denervation and paralysis of the muscles supplied by this nerve. One of the most severe consequences is loss of intrinsic muscle function. They have tingling or complete loss of sensation on the fourth and little fingers. Ulnar nerves seem to be less at risk, possibly because of this unencumbered pathway through the upper arm, providing the opportunity to stretch. Claw-hand is a condition in which the fingers are noticeably curve or bent. This condition can affect one or more fingers, on one or both hands. Claw-hand can be a congenital defect, a defect present at birth, or it may be due to a certain disorders or injuries.

Patient evaluation consists of a focused assessment of upper extremity sense and motor function, radiological studies and most importantly, electrodiagnostic studies [4].

A case of neglected post-traumatic anterior dislocation of the shoulder joint is reported, with an associated fracture of the greater tuberosity and ulnar clawhand, of a perfectly healthy 53-year-old woman.

While the pathoanatomy of deformity will be described in detail here, this condition is due to paralysis of interossei and lumbricals. The claw position of finger with intrinsic paralysis is caused by imbalance between strong extrinsic factors and deficient intrinsic factors. The blocking effect of the transverse lamina on the long extensor will cause metacarpo-phalangeal (MCP) joint that is hyperextended, and increases with further hyperextension, therefore, the long extensor loses its pull on the interphalangeal (IP) joints and allows them to flex 
[4].

\section{Patient Profile}

A 53-year-old woman was involved in falling on her left arm in abductionexternal rotation position on January 2015, she felt discomfort and severe pain (visual analogue scale 7/10) without vascular problems and inability to use the left arm. At that time, deformity of the ring and little fingers known as claw-hand was not recognized. However two months later, she gradually started to feel bent on her ring and little fingers, since the patient had treated herself conservatively. After approximately six months following the injury, she came to our clinic at Dr. Hasan Sadikin Teaching Hospital Bandung to cure her symptoms. On the clinical examination, she demonstrated loss of normal contour of the left shoulder joint, prominence of acromion bone, and slight atrophy of the lateral deltoid muscle (Figure 1). There was a change of the humeral head down in front of the shoulder. There was no atrophy of the shoulder girdle muscles including supraspinatus, infraspinatus and teres minor. There was tenderness over the lateral part of the shoulder.

Movement of the glenohumeral joint was recorded. It was limited in all directions. Besides the limitation of the shoulder, there was an associated neurologic deficit such as numbness and tingling on her left hand especially ring and little fingers in particular on palmar and volar aspect (Figure 2).

Conventional radiological views were taken in one plane anteroposterior (AP)

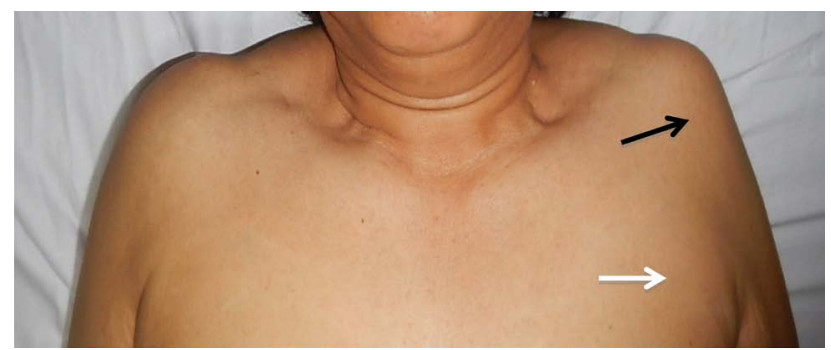

Figure 1. Clinical photograph demonstrating loss of the normal contour of the deltoid (black arrow) of the left shoulder. The humeral head itself may well be palpable anteriorly (white arrow).
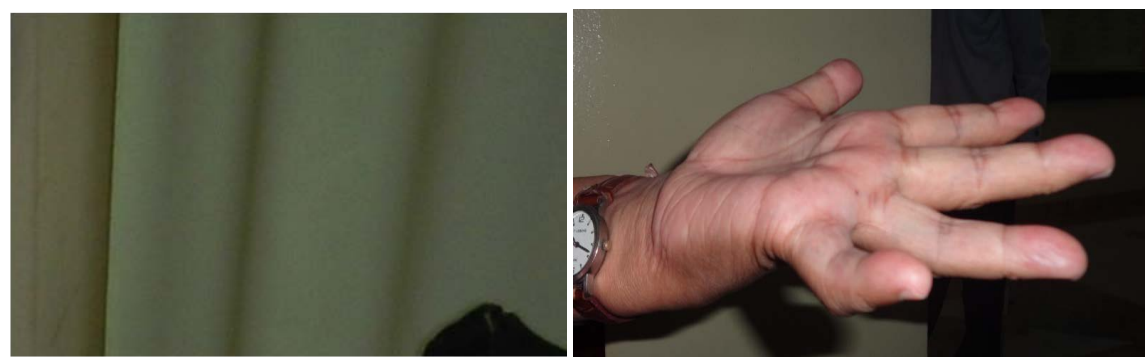

Figure 2. Clinical appearance of left hand who has compression of the ulnar nerve following neglected shoulder dislocation. Before the surgery (left), she had significant clawing of the ring and little fingers and wasting of the intrinsic muscles, and after the surgery (right), she had completely recovered the ring finger, with the exception of the little finger. 


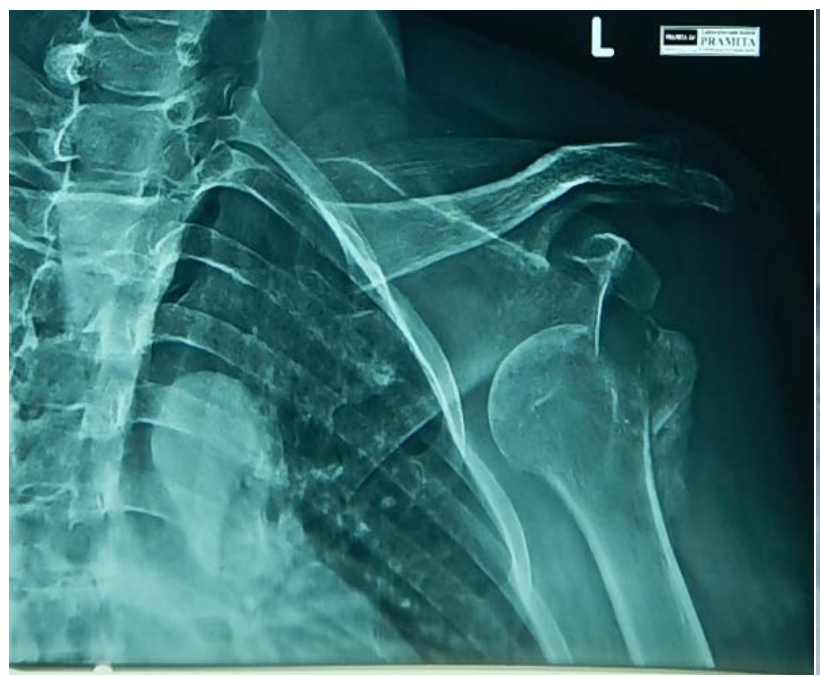

(a)

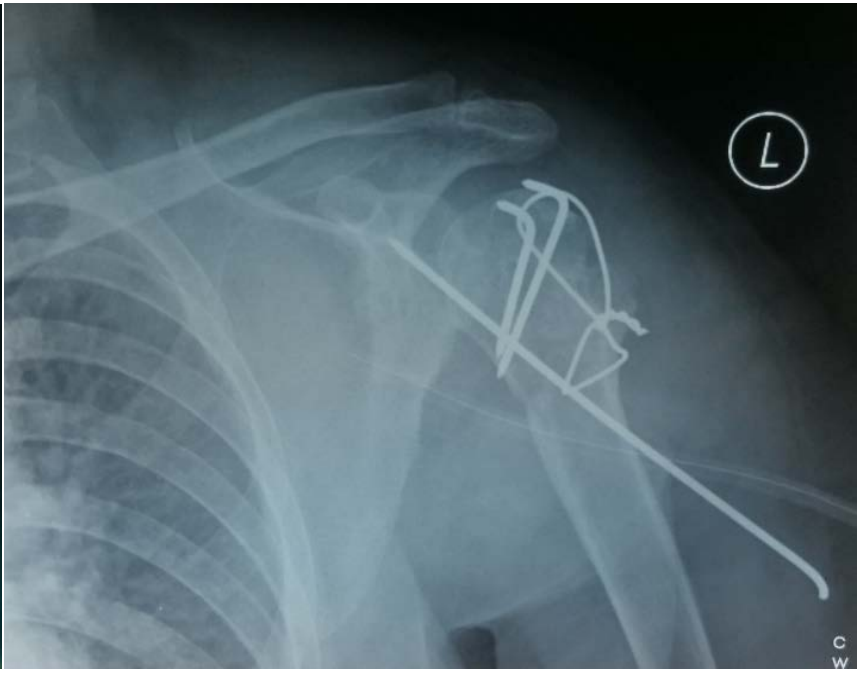

(b)

Figure 3. An AP radiograph of the left shoulder showing anteroinferior dislocation associated with fracture of greater tuberosity (a); Early postoperative AP view showing fixation using a tension band wiring (TBW) and stabilization by a Kirschner wire (b).

only. It revealed glenohumeral joint dislocation (subglenoid type), the humeral head was below the glenoid and associated with fracture of greater tuberosity (Figure 3(a)).

The EMG-NCV examinations were used to determine the severity, the pattern and the recovery of ulnar nerve lesion as a cause of this deformity. Table 2 revealed the result of EMG-NCV before and after surgery.

Surgical procedure was performed by open reduction and internal fixation to reduced humeral head to glenoid and greater tuberosity fracture was reduced to its position and TBW technique was performed to fix the fracture (Figure 3(b)). Intraoperatively findings were dislocation of the humeral head to infraglenoid, fracture of the greater tuberosity and torn of the anterior capsule due to absorption process of the middle and inferior glenohumeral ligaments (MGHL and IGHL). At surgery, the anterior capsule was obliterated and replaced by a rectangular piece of fascia $(2 \times 15 \mathrm{~cm})$ which was harvested from a femoral bone ipsilateral, and inserted on the anterior part of the glenoid and located in two to six o'clock in zig zag position. Transfixing Kirschner wire was used to immobilized the humeral head after reduction (Figure 3(b)) to avoid stretching of the tightened and recontructed soft tissue structures. The Kirschner wire was removed at six-week postoperatively. At the latest follow-up six months postoperatively, the integrity of greater tuberosity and glenohumeral joint was maintained but the ulnar claw-hand still remained slightly in particular in the little finger. However, her numbness and tingling in the ring and little fingers disappeared completely, the bent shape of the ring finger was back to completely normal. Unfortunately, the bent shape of the little finger still remained slightly on the distal interphalangeal joint (DIP) joint, but the PIP joint diappeared completely (Table 1 ).

Moreover, electrodiagnostic study showed it had relieved the ulnar compression, meaning that neurological deficit of the ulnar nerve was recovered partly. 
Table 1. Range of motion on the affected fingers, before and after surgery.

\begin{tabular}{ccc}
\hline & \multicolumn{2}{c}{ ROM of the affected fingers } \\
\cline { 2 - 3 } Affected area & Before surgery & After surgery \\
\hline Ring finger & $45^{\circ}-50^{\circ}$ & $10^{\circ}-100^{\circ}$ \\
the PIP joint & $45^{\circ}-50^{\circ}$ & $5^{\circ}-100^{\circ}$ \\
the DIP joint & $45^{\circ}-50^{\circ}$ & $0^{\circ}-110^{\circ}$ \\
Little finger & $45^{\circ}-50^{\circ}$ & $5^{\circ}-100^{\circ}$ \\
the PIP joint &
\end{tabular}

Table 2. EMG-NCV result of ulnar nerve deficit before and after surgery.

\begin{tabular}{ccc}
\hline & Before surgery & After surgery \\
\hline Ulnar motoric & & \\
Distal onset latency & $4.1 \mathrm{~ms}$ & $3.4 \mathrm{~ms}$ \\
Amplitude & $2.1 \mathrm{mV}$ & $3.9 \mathrm{mV}$ \\
Conduction velocity & $47 \mathrm{~m} / \mathrm{s}$ & $39 \mathrm{~m} / \mathrm{s}$ \\
Ulnar sensory & & \\
Distal onset latency & No response & $4.8 \mathrm{~ms}$ \\
Amplitude & No response & $2.6 \mu \mathrm{V}$ \\
Conduction velocity & No response & $29 \mathrm{~m} / \mathrm{s}$ \\
\hline
\end{tabular}

In this case, EMG evaluation demonstrated the ulnar motoric amplitude and conduction velocity still remained in the condition of before and after surgery, however the ulnar sensory in all parameters (distal onset latency, amplitude and conduction velocity), significantly improved from no response to $29 \mathrm{~m} / \mathrm{s}$ for conduction velocity (Table 2 ).

At six-month follow-up, the patient's condition had clinically improved included the range of motion on the left shoulder. Unfortunately, the X-ray follow-up demonstrated collapse of the humeral head. The upper limb was secured postoperatively according to Rowe and Zarins scoring system. This scoring system evaluates stability, motion and shoulder function [6].

\section{Discussion}

The ulnar nerve originates in the nerve roots of $\mathrm{C} 8$ and $\mathrm{T} 1$ and it is the thickest branch of the medial fascicle of brachial plexus. The ulnar nerve is only affected in $8 \%$ of patients with such nerve injuries, either on its own or in association with other nerve injuries. Similar patterns of damaged ulnar nerve occur in patients with proximal humeral fractures, which are sometimes associated with shoulder dislocation. Pressure on or injury to the ulnar nerve may cause denervation and paralysis of the muscles supplied by the nerve, clinically, experiencing numbness and tingling along the ulnar half of the ring finger and little fingers. Since there is compression on the ulnar nerve, then this will promote increase 
pressure inside the nerve, causing delays in conducting stimuli. This discomfort is often accompanied by weakness of grip and intrinsic wasting [7] [8].

Anterior shoulder dislocations may cause several potential complications. Around $20 \%-50 \%$ of patients will have some types of neurologic complication [8] [9]. Isolated fracture of the greater tuberosity accounts for approximate $20 \%$ of all proximal humeral fracture [10] [11]. They are often associated with dislocation of humeral head antero-inferiorly or can result from an impaction injury, against the lower surface of the acromion or superior glenoid fossa [12].

The treatment is usually open surgery to reduce humeral head to glenoid fossa, and capsular reconstruction, with the use of fascia which harvests from ipsilateral femur, performed to address a deficient anterior aspect of the capsule, by sewing it to the edge of the glenoid fossa. There are several surgical options for unstable shoulder with a deficient capsule. Warner et al. reported a good outcome after using hamstring tendons. Gallie and Le Mesurier described the use of iliotibial band with irreparable capsule, and the results were generally good, with no recurrences [13] [14]. This patient also had greater tuberosity fracture, but it was successfully reduced and fixed by TBW technique [15].

The diagnosis is based on taking a detailed history, making a detailed examination and using appropriate complementary examinations. The mechanism of associated nerve damage is similar to that of the shoulder injury and results in a traction lesion of nerves stretched over the head of the humerus during the dislocation of the head [15]. In the case presented here, the ulnar claw-hand occurred as a result of overstretched ulnar nerve across humeral head since it was dislocated for a considerable time down anteriorly and inferiorly. Together with repeated use of the left upper extremity for around 6 months, the ulnar nerve became gradually stretched.

At six-week follow-up after removing the transfixing wire, the arm was kept anterior to the coronal plane of the body by means of an arm sling. Most reports have recommended crossed transfixing pins through the humeral head into the glenoid fossa for ten to fourteen days to prevent recurrence of dislocation following open reduction [16] [17]. Glenohumeral fixation by Kirschner-wire was used for 4 weeks in this case and the result was evaluated according to Rowe and Zarins system [6] and it yielded good functional results. After reduction patients are generally immobilized for 4 - 6 weeks under sling. The arm support was loosened three times a day to allow early shoulder motion up to $90^{\circ}$ of forward flexion and 10 to 20 degrees external rotation and full elbow motion. After three weeks, flexion and external rotation were gradually increased and with gradual stretching and improving subscapularis contracture, more external rotation was regained. Interestingly, the ulnar claw-hand had gradually disappeared in two weeks after the reduction of this humeral head including numbness and pain as well, meaning that compression and stretching of the ulnar nerve had been released.

In this patient, recovery of shoulder function was incomplete because arthro-fibrotic stiffness was seen. It showed a variable degree of restriction of motion 
in comparison with healthy side. The degenerative changes occurring here in final radiographs make sense because the fracture-dislocation has occurred long enough. Even so, the patient can still perform daily activities without feeling pain.

Literatures described the association between greater tuberosity fracture, dislocation, complete axillary and brachial plexus injury in conjunction with shoulder dislocation [18] [19]. The best means to confirm a concomitant periphery nerve injury with shoulder dislocation includes detailed subjective and objective clinical examinations, along with EMG studies [8]. EMG is the neurological examination of choice for locating the level of compression. In this nerve-conduction study, it shows reduction of the amplitude of the potential and a reduced conduction velocity. In this study, EMG shows little active denervation.

In the case presented here, the patient presented improvement of her pain, tingling and paresthesia, improvement of the EMG and nerve conduction velocity and correction of the ulnar claw. The latter deformity should be treated early by hand physiotherapy until the expectations for recovery are abandoned. This is a rare event in the literature, and early surgical treatment finally, satisfactorily offers a moderate functional EMG-NCV results.

\section{Acknowledgements}

Written informed consent was obtained from the patient for publication of this paper and for the use of any accompanying images.

\section{Conflict of Interest}

The author confirms that this article content has no conflict of interest.

\section{References}

[1] Bankart, A.S.B. (1938) The Pathology and Treatment of Recurrent Dislocations of the Shoulder Joint. British Journal of Surgery, 26, 23-29.

https://doi.org/10.1002/bjs.18002610104

[2] Hovelius, L., Augustini, B.G., Fredin, H., Johansson, O., Norlin, R. and Thorling, J. (1996) Primary Anterior Dislocation of the Shoulder in Young Patients. A Ten-Year Prospective Study. The Journal of Bone and Joint Surgery, 78, 1677-1684. https://doi.org/10.2106/00004623-199611000-00006

[3] Bost, F.C. and Inman, V.C. (1942) The Pathological Changes in Recurrent Dislocation of the Shoulder: A Report of Bankart's Operative Procedure. The Journal of Bone and Joint Surgery, 23, 596-613.

[4] Mulder, J.D. and Landsmeer, J.M.F. (1968) The Mechanism of Claw Finger. The Journal of Bone and Joint Surgery, 50, 664-668.

[5] Aguiar, P.H., Bor-Seng-Shu, E., Gomes-Pinto, F., Almeida-Leme, R.J., Freitas, A.B., Martins, R.S., et al. (2001) Surgical Management of Guyon's Canal Syndrome, an Ulnar Nerve Entrapment at the Wrist: Report of Two Cases. Arquivos de NeuroPsiquiatria, 59, 106-111. https://doi.org/10.1590/S0004-282X2001000100022

[6] Rowe, C.R. and Zarins, B. (1982) Chronic Unreduced Dislocation of the Shoulder. The Journal of Bone and Joint Surgery, 64, 494-505. 
https://doi.org/10.2106/00004623-198264040-00004

[7] Ruas, E.S., Castilho, R.S., Maia, P.E.C. and Melo, G.L.R. (2010) Guyon’s Canal Syndrome Due to a Synovial Cyst. Revista Brasileira Ortopedia, 45, 623-626.

https://doi.org/10.1590/S0102-36162010000600020

[8] Visser, C.P., Coene, L.N., Brand, R. and Tavy, H. (1999) The Incidence of Nerve Injury in Anterior Dislocation of the Shoulder and Its Influence on Functional Recovery. A Prospective Clinical and EMG Study. The Journal of Bone and Joint Surgery, 81, 679-685.

[9] Blorn, S. and Dahback, L.O. (1970) Nerve Injuries in Dislocations of the Shoulder Joint and Fractures of the Neck of the Humerus. Acta Chirurgica Scandinavica, 136, 461-466.

[10] Chun, J.M., Groh, G.I. and Rockwood Jr., C. (1994) Two-Part Fractures of the Proximal Humerus. Journal of Shoulder and Elbow Surgery, 3, 273-287. https://doi.org/10.1016/S1058-2746(09)80071-2

[11] Kim, E., Shin, H.K. and Kim, C.H. (2005) Characteristics of an Isolated Greater Tuberosity Fracture of the Humerus. Journal of Orthopaedic Science, 10, 441-444. https://doi.org/10.1007/s00776-005-0924-6

[12] Court-Brown, C.M., Garg, A. and McQueen, M.M. (2001) The Epidemiology of Proximal Humeral Fractures. Acta Orthopaedica Scandinavica, 72, 365-371. https://doi.org/10.1080/000164701753542023

[13] Millet, P.J., Clavert, P. and Warner, J.P. (2005) Open Operative Treatment for Anterior Shoulder Instability: When and Why. The Journal of Bone and Joint Surgery, 87, 419-432.

[14] Gallie, W.E. and Le Mesurier, A.B. (1948) Recurring Dislocation of the Shoulder. The Journal of Bone and Joint Surgery, 30, 9-18.

[15] Platzer, P., Kutscha-Lissberg, F., Lehr, S., Vecsei, V. and Gaebler, C. (2005) The Influence of Displacement on Shoulder Function in Patients with Minimally Displaced Fractures of the Greater Tuberosity. Injury, 36, 1185-1189. https://doi.org/10.1016/j.injury.2005.02.018

[16] Shin, A.Y., Deitch, M.A., Sachar, K. and Boyer, M.I. (2005) Ulnar-Sided Wrist Pain: Diagnosis and Treatment. Instructional Course Lectures, 54, 115-128.

[17] Milton, G.W. (1953) The Mechanism of Circumflex and Other Nerve Injuries in Dislocation of the Shoulder, and the Possible Mechanism of Nerve Injuries during Reduction of Dislocation. Australian and New Zealand Journal of Surgery, 23, 2530. https://doi.org/10.1111/j.1445-2197.1953.tb05013.x

[18] Wilson, J.C. and McKeever, F.N. (1949) Traumatic Posterior (Retroglenoid) Dislocation of the Humerus. The Journal of Bone and Joint Surgery, 31, 160-172. https://doi.org/10.2106/00004623-194931010-00018

[19] Rockwood, C. and Green, D.P. (1975) Fracture. J.B. Lippincott Company, Philadelphia, 710-718. 
Submit or recommend next manuscript to OALib Journal and we will provide best service for you:

- Publication frequency: Monthly

- 9 subject areas of science, technology and medicine

- Fair and rigorous peer-review system

- Fast publication process

- Article promotion in various social networking sites (LinkedIn, Facebook, Twitter, etc.)

- Maximum dissemination of your research work

Submit Your Paper Online: Click Here to Submit

Or Contact service@oalib.com 